\title{
Microstructural model for cyclic hardening in F-actin networks crosslinked by $\alpha$-actinin
}

\author{
Horacio López-Menéndez ${ }^{\mathrm{a}, \mathrm{b}, *}$, José Félix Rodríguez ${ }^{\mathrm{b}}$ \\ ${ }^{a}$ Cell Adhesion and Mechanics, Institut Jacques Monod (IJM), CNRS UMR 759286 \\ Universitè Paris Diderot, Paris, France \\ ${ }^{b}$ Aragon Institute for Engineering Research (I3A), University of Zaragoza, 50018 \\ Zaragoza, Spain
}

\begin{abstract}
The study of biopolymeric synthetic networks attracts great attention as biomaterials. They can teach us the way to handle this new micro/nano technology as well as contribute to a better understanding of the cytoskeletonlike structural building blocks. A complete understanding of the rheological properties of this novel materials demands the study on extreme situations. Nonlinear deformations can irreversibly alter the mechanical properties of materials. Most soft materials, such as rubber and living tissues, show pronounced softening when are cyclically deformed, this phenomena is recognized as Mullins effect. On the contrary, reconstituted networks of F-actin crosslinked with $\alpha$-actinin harden when are subject to cyclical shear. As a consequence, they exhibit a kind of "mechano-memory" where a significant stress barrier is generated at the maximum of the cyclic shear strain; as was showed by (Schmoller et al., 2010). In this work we propose a microstructural model at the mesoscale, into the framework of nonlinear continuum mechanics. The F-actin filaments are modeled using the wormlike chain model for semiflexible filaments and the intricate gelation process will be introduced as mesoscale crosslinkers dynamics according the physical interactions due to the entanglement and the $\alpha$-actinin. The model is validated with reported experimental results on reconstituted F-actin crosslinked networks with $\alpha$ actinin.
\end{abstract}

\footnotetext{
*Principal corresponding author

Email addresses: horacio.lopez-menendez@ijm.fr (Horacio López-Menéndez), jfrodrig@unizar.es (José Félix Rodríguez)
} 
Keywords: F-actin networks, Mullins-effect, physical crosslinkers, chemical crosslinkers, sacrificial bonds.

\section{Introduction}

Bio-polymeric networks attracts great attention as bio-materials as a result of their soft and wet nature, similar to many biological scaffolding structures. Any given application, however, requires a combination of mechanical properties including stiffness, strength, toughness, damping, fatigue resistance and self-healing. The study of these structures can teach us the way to handle this new micro/nano technology as well as contribute to better understand the cytoskeleton-like structural building blocks, as was shown by Keber et al. (2014). In order to o gain a complete understanding of the rheological properties of this novel materials, it is necessary the study of extreme situations. Nonlinear deformations can irreversibly alter the mechanical properties of materials. Most soft materials, such as rubber and living tissues, show pronounced softening when they are cyclically deformed, this effect is well recognized as Mullins effect (Diani et al., 2009). The material exhibits a mechano-memory where a significant barrier is generated at the maximum of the cyclic shear strain. This unique response is crucially determined by the network architecture. In addition to the normal Mullins effect, some biopolymers networks show an unique cyclic hardening response if they are repeatedly sheared to a certain amplitude. In fact, bundle actin networks not only exhibit softening at low deformation (Gardel et al., 2004), but under certain conditions, they exhibit a drastic increase in the stress needed to reach the maximal applied strain (Schmoller and Bausch, 2013). Importantly, the large mesh size of these biopolymer hydrogels makes them an ideal model system to study the microscopic of such a complex nonlinear response. Indeed, the origin of the observed mechano-memory has been identified by a combination of macrorehology and confocal microscopy, which allowed to visualized the changing network structure during the deformation process (Schmoller et al., 2010; Schmoller and Bausch, 2013).

The idea that the cytoskeleton and cytoskeleton-like materials are passive structures that allows an active behavior only under the action of the actinmyosin molecular motors is currently under discussion (Sun et al., 2010; Walcott and Sun, 2010). What seems to be right for sarcomeric cells, like heart or muscle cells, seems to be different for non sarcomeric cells where the cystoskeleton undergoes a constant remodeling process (Howard, 2001; Trepat 
et al., 2007; Phillips et al., 2009). In this regard, in-vitro actin networks without active molecular motors have been considered to be in thermodynamic equilibrium (Mizuno et al., 2008). However, experiments performed by Schmoller et al. (2010); Lieleg et al. (2011) on artificially reconstructed F-actin networks have shown that this networks are non-equilibrium networks. In these experiments Schmoller et al. (2010) have observed that the network is able to build up kinetically trapped stress during network formation providing these networks with a rather unique behavior. Differently to most soft materials, such as rubber and living soft tissues, where nonlinear deformations irreversibly alter the mechanical properties of the material by causing a pronounced softening when they are cyclically deformed, reconstituted crosslinked actin networks show softening and hardening effects when the network is subject to cyclic shear strain (Schmoller et al., 2008). This kind of networks have an intricate gelation process, where the interaction between the actin filaments and the actin-binding-proteins (ABP's), given by $\alpha$-actinin in this case, are rather weak (in the order of few $k_{B} T$ ). Therefore, even without an external thermodynamic driving force, the bonds are binding and unbinding at physiological temperature (Gardel et al., 2004; Lieleg et al., 2010, 2011; Schmoller et al., 2008, 2009, 2010; Wolff et al., 2010, 2012).

Developing constitutive models for artificially reconstructed crosslinked F-actin networks can help to understand the role of the F-actin network in the cell cytoskeleton (Heussinger et al., 2007). Wolff et al. (2010, 2012) develop a formalism called iGWLC (inelastic glassy wormlike chain) to link the nonlinear mechanical description of the wormlike chain model with the dynamic of crosslinkers. This model describes the experimental results of observed effect of stiffening and softening, which can be considered as a description of the Mullins effect in actin networks, considering the crosslinkers dynamics. The developed solution was done in the Fourier domain, which is suitable for the experimental description of rheological experiments. Others models developed by (Van Dillen et al., 2008; Kim and Sun, 2009; Kim et al., 2009; Abhilash et al., 2012; Cyron et al., 2013) using computational techniques based on the reconstruction of the networks by means different methods such as brownian dynamics and finite element; the interaction between filaments where described by the dynamics of crosslinkers. These models have a strong demand of computational resources in order to gain information about the rheological properties.

At tissue and rubber scale, several models have been developed for the standard Mullins effect. Usually, the description of the constitutive behavior 
of this type of material relies on the identification of an appropriate strainenergy density function (SEF) from which stress-strain relations and local elasticity tensors can be derived (Holzapfel, 2000). A number of SEF has been proposed to describe the behavior of soft tissue with damage. These models are based on the introduction of internal variables that account for non-physiological loading that drives soft tissue to damage. In most models, the main damage mechanism is associated with tear or plastic deformation of fibers. Hurschler et al. (1997) proposed a micromechanical model for ligament behavior that includes fiber failure. Similarly, we found the model of Arnoux et al. (2002) and Schechtman and Bader (2002) for ligaments and tendons, or the work of Hokanson and Yazdami (1997) for damaging arteries. Gasser and Holzapfel (2002) proposed a rate-independent multisurface elastoplastic constitutive model for soft tissue which introduced inelastic deformation of the collagenous component of the tissue. Balzani et al. (2006) proposed a discontinuous damage model for arteries in which the damage of the fibers is treated following classical continuous damage theory. Also, Rodriguez et al. (2006) developed a constitutive model which accounts for different damage processes for matrix and fibers. Fibrous part was assumed to follow the wormlike chain model (Mackintosh et al., 1995) where damage was incorporated through the statistical distribution of the deformation at the fully extended length of collagen fiber bundles (crosslinks rupture). More recently Saez et al. (2012) have proposed a microsphere based model for modeling damage in fibrous tissue where the directional statistics is used to describe the orientation of collagen fibbers within the tissue.

This paper proposes a formalism within the framework of nonlinear continuum mechanics which introduces dynamics of gelation by means of a mesoscale model of the interaction between the network and the crosslinkers. In the next section we describe the nonlinear mechanics of semiflexible filaments from the perspective of continuum mechanics. After that we will introduce the complexity of the gelation process considering the effects of physical and chemical crosslinkers and the interactions between them and with the fiber considering simple arguments from the physics of polymers. Then, we will arrive to a mesoscale description of the F-actin network considering the dynamics of the gelation with few free parameters easy to adjust. Afterwards, we will describe the monotonic and cyclic the experiments, showing an opposite to the expected Mullins effect, and the study of the evolution of the free parameters during the cyclic process which will give to us some ideas regarding the evolution of the microstructure through the experiment. 


\section{Materials and methods}

Filament elasticity will be described using the wormlike chain model in the form proposed by Mackintosh et al. (1995) and further developed by Palmer and Boyce (2008), whereas the network will be described using an homogenized continuum framework based on the eight chain network with rigid crosslinkers (Arruda and Boyce, 1993; Bertoldi and Boyce, 2007; Palmer and Boyce, 2008; Brown et al., 2009). We will take as starting point the mechanics of networks with rigid crosslinkers and introduce the inelastic effect as alterations in the contour length of the F-actin network. To define the phenomenological law that drives the changes in the contour length we will propose a simple model for the gelation process of the network based on the interactions between the physical and chemical crosslinkers.

\subsection{Entropic bundle network elasticity}

Mechanical behavior of single actin filaments is governed by the worm-like chain $(w l c)$ model for semiflexible filaments, as was proposed by Mackintosh et al. (1995) to describe crosslinked polymer networks in which the forcestretch relationship is given by

$$
F_{w l c}=\frac{k_{B} T}{l_{p}}\left[\frac{1}{4\left(1-\frac{r}{L_{c}}\right)^{2}}\right]\left[\frac{\frac{L_{c}}{l_{p}}-6\left(1-\frac{r}{L_{c}}\right)}{\frac{L_{c}}{l_{p}}-2\left(1-\frac{r}{L_{c}}\right)}\right],
$$

where $k_{B}$ is the Boltzmann constant, $T$ is the absolute temperature, $L_{c}$ is the bundle length, $l_{p}$ is the persistence length, and $r$ represents the end-to-end distance (see Fig. 1a). The persistence length is defined as the length at which entropic contributions to elasticity become important, as the bundle shows significant bending purely due to its thermal energy. A bundle with $L_{c}>>l_{p}$ will bend, even without application of forces. In order to extend the model in Eq. (1) from a single filament to a continuum description of the F-actin network, we adopt the approach proposed by Palmer and Boyce (2008) based on the eight-chain model. In this model, the network, assumed to be isotropic, is idealized as an unit cube with eight chains, or bundles, extending from the center to each of the vertices of the cube (see Fig. 1b). The reference (undeformed) end-to-end distance of each bundle is $r_{0}$, so that $r_{0}=\sqrt{3} / 2$. Due to applied stress the unit cube becomes a cuboid in the 
deformed configuration. If the unit cube is aligned with the principal stretch directions, it can be shown that the stretch of any chain in the unit cube, $\lambda$, is given by Palmer and Boyce (2008)

$$
\lambda=\frac{r}{r_{0}}=\sqrt{I_{1} / 3}
$$

where $I_{1}$ is the first invariant of the right Cauchy-Green deformation tensor $\mathbf{C}=\mathbf{F}^{T} \mathbf{F}$, and $\mathbf{F}=\partial \mathbf{x} / \partial \mathbf{X}$ is the deformation gradient, where $\mathbf{x}$ is the position of a material point in the current configuration and $\mathbf{X}$ is the original position. Therefore, the end-to-end distance $r$ can now be written as $r=$ $r_{0} \sqrt{I_{1} / 3}$, and the force stretched relation in Eq. (1) expressed in terms of the deformation tensor $\mathbf{C}$.

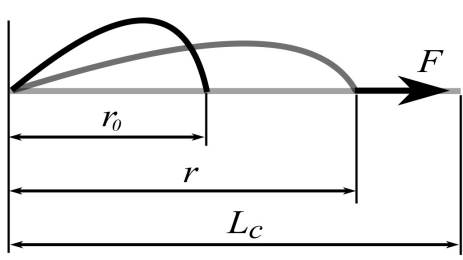

(a)

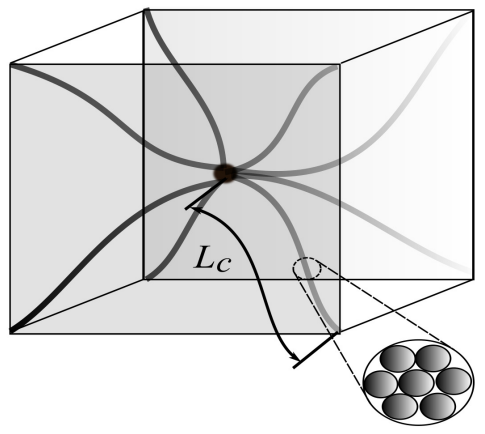

(b)

Figure 1: a) Single filament schematic and b) Idealized eight chain model of an F-Actin network.

From a continuum mechanics point of view, it is convenient to identify a strain energy density function for the network. This can be achieved by calculating the work done by each chain (integrating the filament force-extension expression in Eq. (1)) and then multiplying the resulting expression by the filament density, $n$ (number of filaments per unit volume). Following the procedure proposed in Palmer and Boyce (2008) we obtain

$$
\psi_{w l c}=\frac{n k_{B} T}{l_{p}}\left[\frac{L_{c}}{4\left(1-\frac{r}{L_{c}}\right)}-l_{p} \log \frac{L_{c}^{2}-2 l_{p} L_{c}+2 l_{p} r}{r-L_{c}}-c\right]
$$


where $c$ is a constant equal to the initial strain energy density from the filaments. Since the F-Actin network is embedded in a nearly-incompressible fluid, the strain energy function of the network, $\Psi_{w l c}$, is rewritten as

$$
\Psi_{w l c}\left(\mathbf{C}, l_{p}, L_{c}\right)=\psi_{w l c}\left(r, l_{p}, L_{c}\right)+p(J-1),
$$

where, $\Psi_{w l c}$ is defined for $J=\operatorname{det} \mathbf{F}=1$. The scalar $p$ is an indeterminate Lagrange multiplier which can be identified as a hydrostatic pressure, and that is obtained from the equilibrium equations and boundary conditions.

Using standard procedures from Continuum Mechanics, the Cauchy stress, $\boldsymbol{\sigma}$, can be derived from direct differentiation of Eq. (4) with respect to C (Holzapfel, 2000)

$$
\begin{aligned}
\boldsymbol{\sigma} & =\frac{2}{J} \mathbf{F} \frac{\partial \Psi_{w l c}}{\partial \mathbf{C}} \mathbf{F}^{T} \\
& =\frac{n k_{B} T}{3 l_{p}} \frac{r_{0}}{\lambda}\left[\frac{1}{4\left(1-\frac{\lambda r_{0}}{L_{c}}\right)^{2}}\right]\left[\frac{\frac{L_{c}}{l_{p}}-6\left(1-\frac{\lambda r_{0}}{L_{c}}\right)}{\frac{L_{c}}{l_{p}}-2\left(1-\frac{\lambda r_{0}}{L_{c}}\right)}\right] \mathbf{b}+p \mathbf{I}
\end{aligned}
$$

where $\mathbf{b}=\mathbf{F F}^{T}$ is the left Cauchy-Green deformation tensor, and $\mathbf{I}$ is the second order identity tensor.

As mentioned before, the F-actin network is assumed as an out of equilibrium network. If we consider the effects of the kinetically trapped stress over the network structure we should consider that the end-to-end distance in the reference configuration does not correspond to the distance for zero force. For the Mackintosh model, Eq. (1), the expression for the end-to-end distance at zero force is $r_{0_{F=0}}=L_{c}\left(1-\frac{L_{c}}{6 l_{p}}\right)$. In order to describe the prestressed network we introduce an internal variable $\epsilon$ which represents the degree of prestress as a fraction of $r_{0_{F=0}}$, as proposed by Palmer and Boyce (2008). Therefore the expression for $r$ in the out of equilibrium network will be

$$
r=\lambda(1+\epsilon) L_{c}\left(1-\frac{L_{c}}{6 l_{p}}\right)
$$

\subsection{Gelation process}

The experiment performed by Schmoller represents a network with an intricate gelation process, where the rheological response will be strongly dependent upon the conditions of the preparation Witten and Pincus (2010). 
The joint interaction between $\alpha$-actinin, dense fraction of semi-flexible filaments, polymerization, branching, fluctuations, and entanglement will freeze the state of the network in a highly pre-stressed condition and generally in a metastable equilibrium (Lieleg et al., 2011, 2009). To deal with this complicated scenario, we will try to build up a simplistic picture based on the interactions between the physical crosslinkers, chemical crosslinkers and the interaction between them with the semiflexible network structure, see Figure 2. For the general description of the gelation process we will follow similar arguments as those exposed in classical polymers physics bibliography as De Gennes (1979); Witten and Pincus (2010).
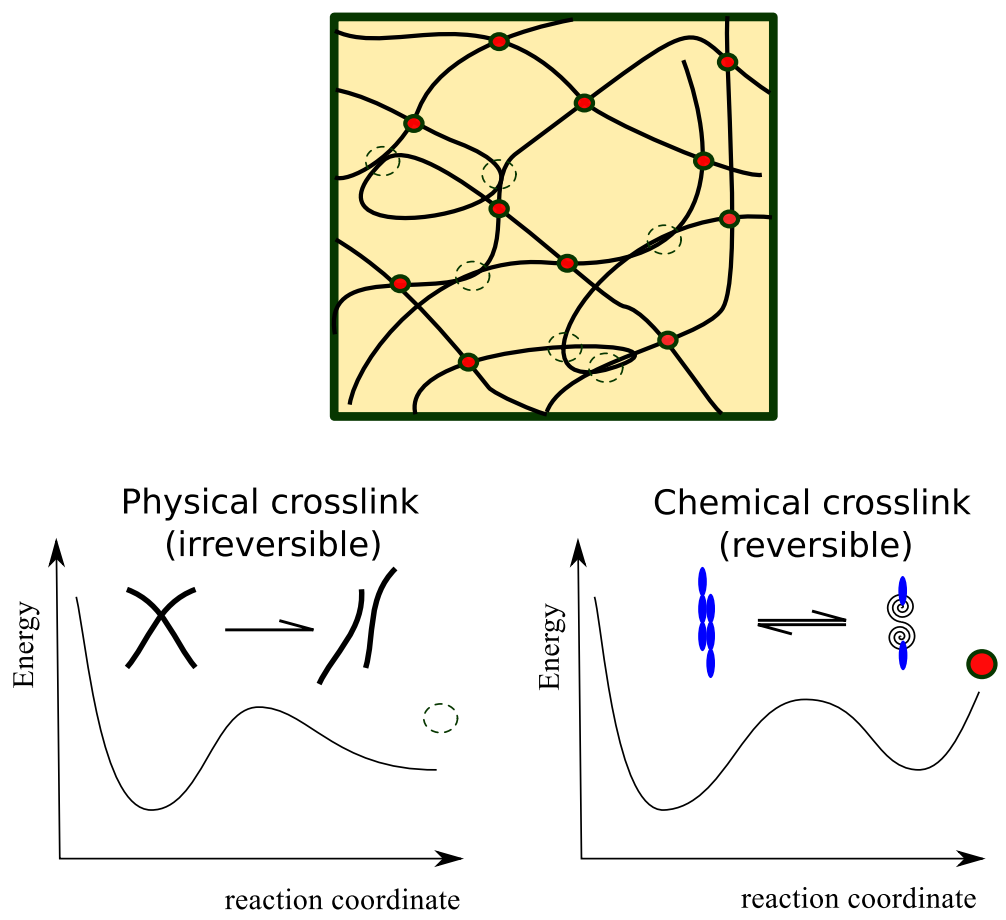

Figure 2: A simplified picture of the network will be made by the combination between semi-flexible crosslinkers, chemical crosslikers, and physical crosslinkers. The chemical crosslinkers will be given by the $\alpha$-actin, whose will develop reversible interaction; and the physical crosslikers, due to the entanglement between filaments, will show an irreversible energy gap.

In the following subsections we will describe the mathematical formulation for the physical and chemical crosslinks, we will also develop the idea about the regulation of the adhesion energy due to the interaction among 
this two kinds of crosslinkers

\subsubsection{Chemical crosslinks}

The chemical crosslinks are given by the $\alpha$-actinin, if they are stable (for the stress and the time scales of the experiments), they provide a strong gelation process. If the crosslinks are not completely stable but are associated with a reaction that can proceed in both directions, as binding-unbinding of the crosslink, we speak of a weak gelation process, and we expect to find some of the intricacies of glass transitions (Witten and Pincus, 2010; De Gennes, 1979). In a simplified way, the chemical crosslinks can be modeled as a reversible two-state equilibrium process (Brown et al., 2009; Purohit et al., 2011); as can be seen in the following expression:

$$
\frac{P_{u b}}{P_{b}}=\exp \frac{-\left(\Delta G_{0}+w_{e x t}\right)}{k_{B} T}
$$

Where $P_{u b}$ defines the unbinding probability encompassing the states of unbinding, unfolding or flexible cross-link, and $P_{b}$ the binding probability encompassing the states of binding, folding or rigid cross-link. Since only these two states are possible, then $P_{u b}+P_{b}=1$. The fractions of each state will written as a function of the deformation energy over them. Our twostate model will have the binded $\alpha$-actinin as the (preferred) low free energy equilibrium state at zero force and the unbinded $\alpha$-actinin as the high free energy equilibrium state at zero force. Let the difference in the free-energy between these states be $\Delta G_{0}$ and the separation between the energy wells corresponding to these be $w_{\text {ext }}$. $k_{B} T$ represents the thermal energy.

Also, if we consider the conservation of probability, we could obtain an expression for $P_{u b}$ as:

$$
P_{u b}=\frac{1}{1+\exp \left[\frac{\left(\Delta G_{0}-w_{e x t}\right)}{k_{B} T}\right]}
$$

\subsubsection{Physical crosslinks and sacrificial bonds}

Any physical process which favors the association between certain points on different chains may lead to gels. The entanglement effect may drive a number of phenomena such as, the formation of helical structures, microcrystals, loops, and also electrostatic, hydrophobic, dipole-dipole interactions (De Gennes, 1979). This is not an equilibrium process, but it corresponds to 
the progressive freezing of a number of degrees of freedom of the bundles via sticky interactions as can be observed in Figure 2.

The physical crosslinkers can create networks with different kinds of stiffness according to the degree of entanglement. In this work we will consider that the physical crosslinkers will develop the role of sacrificial bonds and hidden length as was proposed by Fantner et al. (2005); Buehler (2007); Ducrot et al. (2014). Sacrificial bonds in our case are defined as physical crosslinkers that break themselves, in a fragile way, before the chemical crosslikers of $\alpha$-actinin were broken. The hidden length is defined as the part of the molecule that was constrained from stretching by the sacrificial bond. This mechanism contributes with the toughness of the network by means the relaxation of stress and with the increment of the average contour length. We will consider that the fraction of energy released due to the fragile breakage of physical crosslinks, working as sacrificial bonds, will be dependent of the maximum level of deformation exerted over the network. In other words, just during the first shearing cycle, the sacrificial bonds will break.

In order to describe the probability of fracture for the physical crosslink, we propose an Arrhenius-like relation, in a similar way as was proposed by Bell (1978); Evans (2001); Bertoldi and Boyce (2007); Buehler (2007); Ciarletta et al. (2008), but our phenomenological description will employ the bundle stretch as a driving variable. In this model, the probability of failure will be

$$
P_{f}=P_{f_{0}} \exp \left[\kappa^{f}\left(\lambda_{\max }-\lambda_{0}^{f}\right)\right],
$$

where $\kappa^{f}, \lambda_{0}^{f}$ are mesoscopic material parameter associated with the activation energy needed to break the bond; $\lambda_{\max }$ represent the maximum stretch achieved by the bundle. $P_{f_{0}}$ represents the irreversible bond rupture at $\lambda_{\max }=\lambda f_{0}$.

\subsubsection{Interaction between physical and chemical crosslinkers}

During the gelation process the physical crosslinkers are created by the network entanglement. This process will induce pre-stress across the network, and will be propagated through the bundles until the chemical crosslinkers (Lieleg et al., 2009, 2011). The Figure 3.a illustrates this idea, where the interrupted line describes the physical crosslink, and the red dots represent the chemical crosslinkers. In this configuration the pre-stress will be higher, the contour length $(L c)$ will be lower due to the connectivity introduced by the entanglement. Therefore, the trapped stress into the structure will be 
compensated by the bundle deformation and by the chemical crosslinkers deformation. In consequence, potentially it will be able to induce conformational changes over the $\alpha$-actinin structure, as was described by Golji et al. (2009).
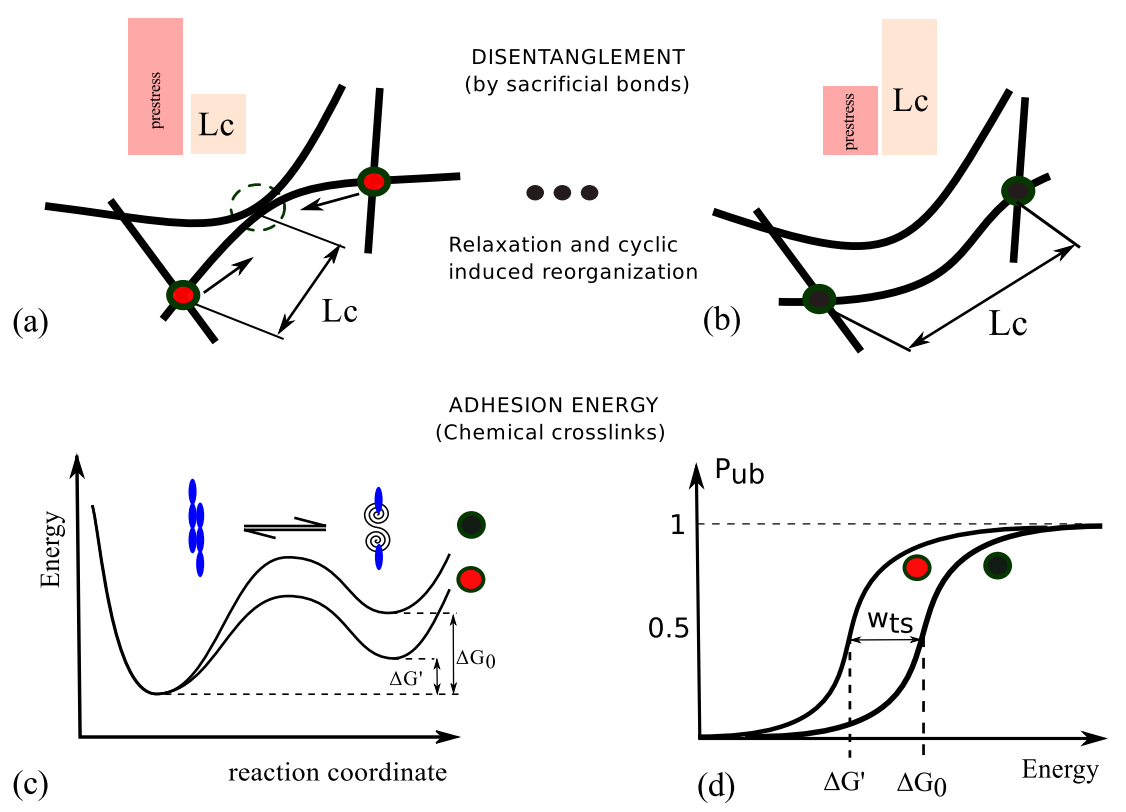

Figure 3: Interaction between physical and chemical crosslinkers. (a) Gelation state in the reference configuration. The physical crosslinks induce prestrain over the bundles and reduce the contour length. The red dots represents a lower adhesion energy state of the $\alpha$-actinin. (b) Once the physical crosslinkers, working as sacrificial bonds, release the energy, increase the contour length, reduce the prestrain over the $\alpha$-actitn, and increase the adhesion energy. The black dots represent a higher adhesion energy of the $\alpha$-actinin. (c) Two energy landscape for the chemical crosslinkers; with and without considering the effect of prestrain imposed the physical crosslinks. (d) Cumulative distribution function for the unbinding probability, $P_{u b}$. The distribution without pre-strain shows a higher transition point.

Therefore, when the network is sheared at certain critical strain, some physical crosslinkers will be more fragile-like and easy to break (those that behave as a sacrificial bonds). Then, some of the trapped stress will be relaxed and redistributed by means the disentanglement driven by the cyclic protocol. As can be observed in the Figure 3.b after the network reorganization, the pre-stress over the bundles and over the crosslinkers will be smaller, and the contour length $(L c)$ will be higher. 
In the Figure 3.c the black dot represents the chemical crosslinker in a more stable state; due to the conformational change introduced by the prestress of the network will be less, and the adhesion energy will increase, changing from $\Delta G^{\prime}$ to $\Delta G_{0}$, with $\Delta G_{0}>\Delta G^{\prime}$.

In order to describe more quantitatively this fact, we will modify the Eq.10 considering the role of the deformation energy exerted over the $\alpha$-actinin structure. This can be understood as a combined action of two mechanical regulation pathways over the $\alpha$-actinin reaction, where: $i) w_{\text {ext }}$ represents the mechanical work induced by the macroscopic deformation that propagates through the network down to the $\alpha$-actinin. $i i) w_{t s}$ represents the mechanical work introduced during the entanglement and the physical crosslinking which also deforms the $\alpha$-actinin structure. If we reorganize the terms defining $\Delta G^{\prime}=\Delta G_{0}-w_{t s_{i}}$, the next expression can obtained:

$$
P_{u b}=\frac{1}{1+\exp \left[\frac{\left(\Delta G^{\prime}-w_{e x t}\right)}{k_{B} T}\right]}
$$

The Figure 3.d illustrates the shifting effect due to the change introduced by $w_{t s}$. This tell us that the adhesion energy will change according to the state of the out-of-equilibrium forces into the network. Therefore, for the same macroscopic strain we will observe different transition points, according to the internal pre-strain of the structure, and as consequence it will explores different energy landscapes.

As we mentioned for the physical crosslinker, the experiments are in the mesoscale, where we are only able to measure macroscopic quantities as stress and strain. Since we are aiming to develop a constitutive model in the mesoscale, we will propose the next phenomenological expression, using the previous expression as motivation:

$$
P_{u b}=\frac{1}{1+\exp \left[\kappa^{u b}\left(\lambda_{0}^{u b}-\lambda\right)\right]},
$$

where the main driving force will be $\lambda$, the average stretch over the bundle and proportional to the macroscopic shear strain. In order to simplify the mathematical treatment, we will consider a linear relationship to approach the deformation energy of the $\alpha$-actinin crosslinker as $\kappa^{u b} \lambda$. Also, $\kappa^{u b} \lambda_{0}$ will be proportional to the intermediate adhesion energy $\Delta G^{\prime}$. Then $\kappa^{u b}$ will give us an idea of the sharpness of the transition between states and $\lambda_{0}$ will be the strain at which the transition is 0.5 . If $\lambda_{0}<<\lambda$ the network will be easy 
to remodel showing a behavior more fluid-like. If $\lambda_{0}>\lambda$ the crosslinker stability will be higher and the probability of transition will be very low, and behaving as solid-like structure.

\subsubsection{Gelation and contour length}

Based on the previously described mechanism we propose the ansatz, Eq.(12), for the average bundle length into the network. This can be considered as a stochastic variable dependent on the irreversible bound rupture probability (physical crosslinkers) and on the reversible unbinding probability (chemical crosslinkers). As can be observed schematically in the Figure 4 .

$$
L_{c}=L_{c}^{f} P_{f}+L_{c}^{u b} P_{u b},
$$

where parameters $L_{c}^{f}$ and $L_{c}^{u b}$ are regarded as material parameters determined from experiments.

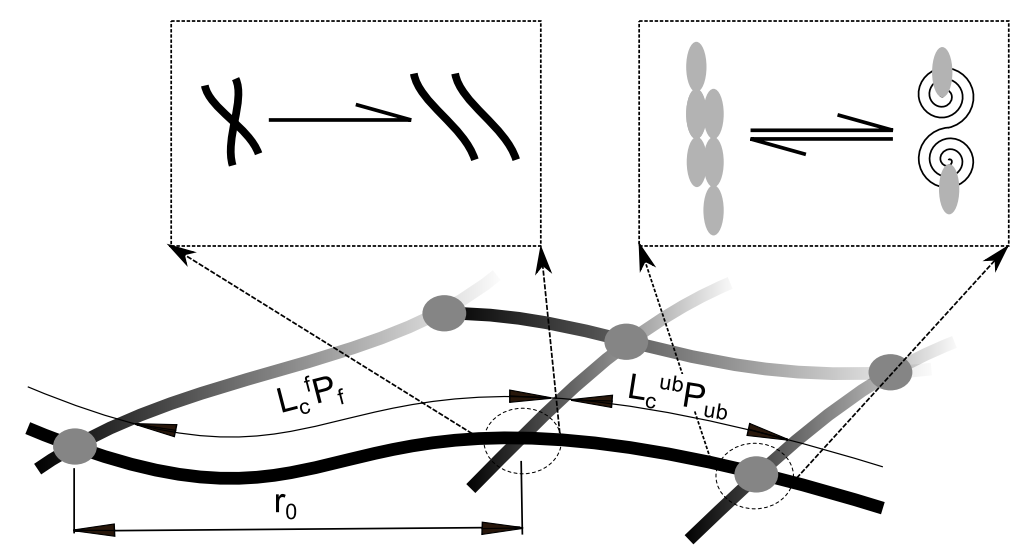

Figure 4: Semiflexible bundle structure and its interaction with the physical and chemical crosslinkers.

\section{Results}

The proposed theory is used to describe the experiments conducted by Schmoller et al. (2010) on artificially reconstituted F-actin networks crosslinked with $\alpha$-actinin; where the network have an actin concentration of $c_{a}=4.75 \mu \mathrm{M}$ and a molar ratio of cross linking molecules to actin, $R=1$, at $18{ }^{\circ} \mathrm{C}$. For large enough concentrations of the cross linker, these networks show a pronounced nonlinear mechanical response to shear strain. These 
networks also show profound network reorganization when is subjected to cyclic shearing.

If we apply a monotonic, and constant shear deformation rate, the network stiffening response start at low levels of strain and will continues almost linearly until reaching a maximum critical shear stress. After that point the stress decreases until reaching a plateau phase that slowly decreases towards zero as the shear strain increases. However, if the network is subject to a cyclic shear strain $\gamma$ applied at a rate of $1.4 \% \mathrm{~s}^{-1}$, and always reaching the same maximum shear strain value, the network experiences a significantly different response from the first cycle. Schmoller et al. observed that, after the first loading cycle, each repetition of the deformation resulted in an increasingly larger linear regime, but also in a network that could withstand much higher stress. This result is in sharp contrast to the Mullins effect observed in rubber-like materials. However, this particular behavior was also found to be very much dependent on the concentration of crosslinkers.

In order to described the previous experiments, the model has been specialized for a pure shear experiment. Therefore, in terms of the shear deformation, $\gamma$, the bundle stretch is given by $\lambda=\sqrt{1+\gamma^{2} / 3}$. The Cauchy shear stress-strain relationship and the remaining equations of the model reduce to:

$$
\begin{gathered}
\tau=\frac{n k_{B} T}{3 l_{p}} \frac{r_{0}}{\lambda}\left[\frac{1}{4\left(1-\frac{\lambda r_{0}}{L_{c}}\right)^{2}}\right]\left[\frac{\frac{L_{c}}{l_{p}}-6\left(1-\frac{\lambda r_{0}}{L_{c}}\right)}{\frac{L_{c}}{l_{p}}-2\left(1-\frac{\lambda r_{0}}{L_{c}}\right)}\right] \gamma, \\
r=\lambda\left(1+\epsilon_{i}\right) L_{c}\left(1-\frac{L_{c}}{6 l_{p}}\right), \\
L_{c}=L_{c}^{f} \exp \left[\kappa^{f}\left(\lambda_{\max }-\lambda_{0}^{f}\right)\right]+\frac{L_{c}^{u b}}{1+\exp \left[\kappa_{i}^{u b}\left(\lambda_{0_{i}}^{u b}-\lambda\right)\right]} .
\end{gathered}
$$

As can be seen, we arrive to a compact set of coupled equations where only three parameters, indicated with the subindex $i$, will change during the cyclic experiment.

\subsection{On the parameters of the model}

There are two kinds parameters into the coupled set of equations. At one side the typical values for the semiflexible-wlc model with rigid crosslinkers 
as $\left(L_{c}, l_{p}, \epsilon, n\right)$. The plausible values for the orders of magnitude can be easily found in the literature as in Gardel et al. (2004); Palmer and Boyce (2008); Lieleg et al. (2010). More specifically the density of actin filaments, $n$, represents a proportionality factor, and it was adopted from Palmer and Boyce (2008). The persistence length $l_{p}$, was taken as 17.48 $\mu \mathrm{m}$ (Gardel et al., 2004). We should point out that the persistence length is also dependent on the crosslinker concentration and loading (Gardel et al., 2004; Lieleg et al., 2010) and should be defined as a stochastic variable as well. However, in order to simplify the model, we will consider $l_{p}$ as a constant parameter in the following. The contour length contribution $\left(L_{c}^{f}, L_{c}^{u b}\right)$ where estimated in the range of values of $L_{c}$ described in the experiment of Schmoller et al. (2010).

On the other side, according to this model, the parameters associated with crosslinker dynamic will encode the transitions whose induce remodeling into the network. The parameters $\left(\kappa_{i}^{u b}, \lambda_{0_{i}}^{u b}\right)$, will change during the cyclic experiment. This values represent an indirect measure of the adhesion energy

of chemical crosslinks of $\alpha$-actinin, $\lambda_{0}^{u b}$ describe the transition point in the contour length of network filament, and $\kappa^{u b}$ the sharpness of this transition, this will provide a fine tuning of the data. The parameters $\left(\kappa^{f}, \lambda_{0}^{f}\right)$, will not evolve during the cyclic parameter due to they depend on the maximum level of strain. They represent the failure dynamics of the physical crosslinkes and its role will be more dominant during the regime of large deformations. In order to avoid the re-stiffening, and guarantee the network softening we should keep the relation $\lambda r_{0}<<L c$.

\subsection{Simulation of the monotonic and cyclic loading experiments}

The monotonic experiment, see Figure 5, shows the results of the model (solid line) along with the experimental data from Schmoller et al. (2010) (black crosses) for a monotonic loading experiment in which the network has been sheared up to a maximum shear strain, $\gamma_{0}=1.2$, at a strain rate of $1.4 \% \mathrm{~s}^{-1}$ (see inset in Figure 5). The model predicts strain hardening response to start at $\gamma \approx 0.1$ and continues until reaching a maximum shear stress $\tau_{\text {max }} \approx 9 P a$ at $\gamma \approx 0.28$ after which the stress decreases until reaching a plateau phase that slowly decreases towards zero as the shear strain increases. For strains lower than 0.4 the model closely follows the experimental data. For larger shear strains, however, the model predictions are constantly biassed from the experimental data. 


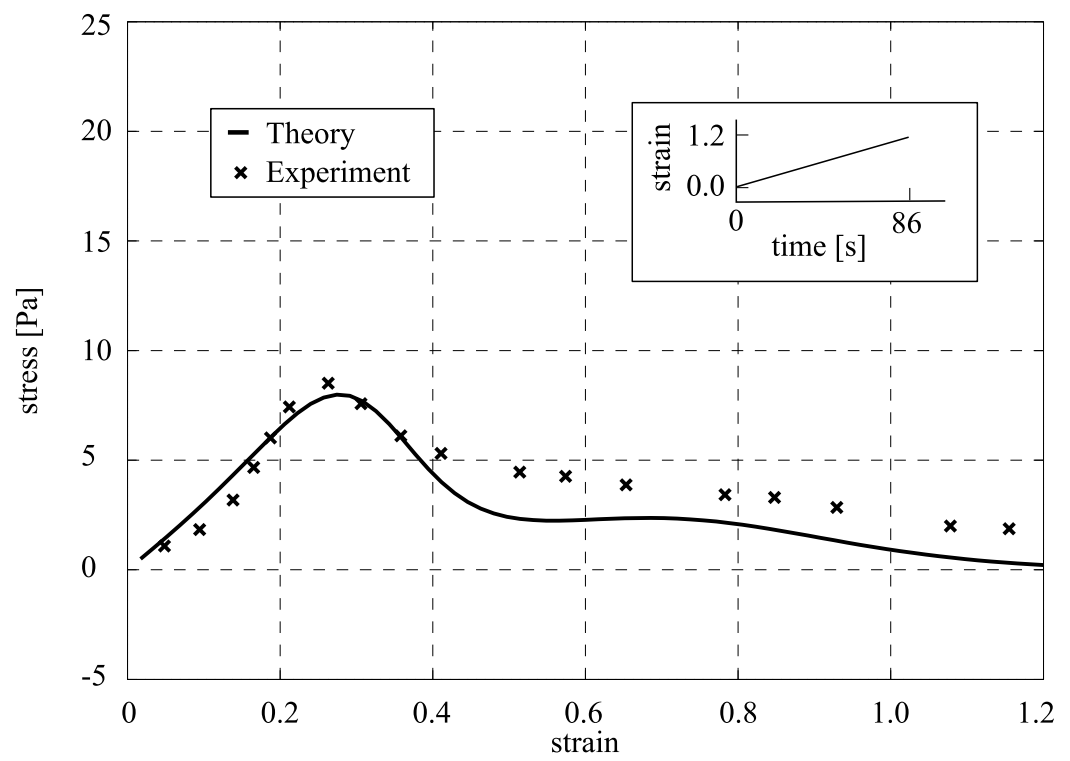

Figure 5: Monotonic shear experiment with maximum shear strain, $\gamma_{0}=1.2$. The figure show the effect of softening of the network associated with the crosslinkers unbinding.

The identified model parameters obtained by best fitting of the experimental data are summarized in Table 1 (see Apendix). Identified parameters are in good agreement with those found in the literature. Palmer and Boyce (2008) found a boundle prestrain of $3 \%$ with F-actin networks with lower actin/crosslinker concentration ratio $(\mathrm{R}=0.03$ and $\mathrm{R}=0.5)$. The initial contour length for our model $(9 \mu \mathrm{m})$ is in good agreement with the mean mesh size of the network reported by Schmoller et al. (2010).

During the cyclic loading protocol the response of the network is very different to the observed for monotonic loading protocol. In the case of cyclic loading, see the experimental measurements in Figure 6, after each strain cycle the linear regime gets larger whereas the network is able to withstand a higher maximum stress, in sharp contrast to the Mullins effect observed in rubber-like materials. When we apply the proposed model, exploring the parametric space for $\kappa_{i}^{u b}, \lambda_{0_{i}}^{u b}$ and $\epsilon^{i}$, we found that is able to fit quite well the experimental measurements of Schmoller, as can be observed in Figure 6 . Evolving the three parameters for each cycles, can be seem how the linear response of the network becomes larger, and how the network is able to reach a larger stress, as a network with rigid crosslinkers (see Table 2 in Apendix). 


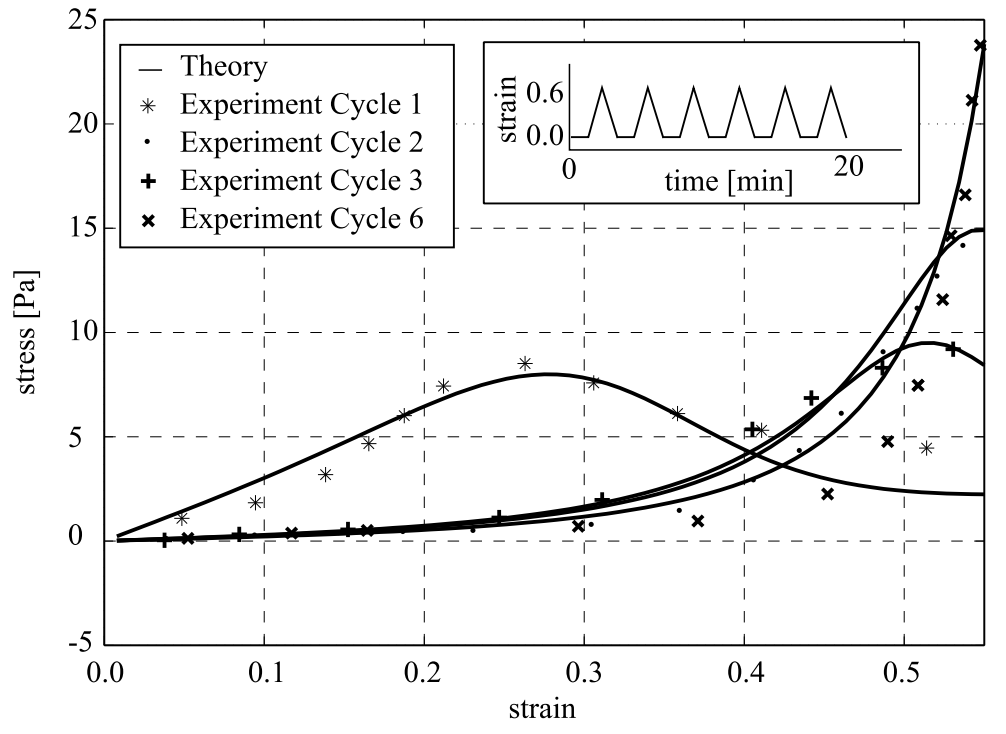

Figure 6: Cyclically sheared with $\gamma_{0}=0.55$. The figure show the effect of hardening of the network as the number of cycles increase

To understand the effects behind the differents set of parameters used to fit the data, the Figure 7 shows the relation between the bundle prestrain and the mesoscale approximation for the adhesion of crosslinkers, $\kappa_{i}^{u b} \lambda_{0_{i}}^{u b}$. The figure demonstrates that the pre-strain decreases monotonically with the number of cycles whereas the adhesion of crosslinkers increases indicating the stabilization of the network. To describe more quantitative, we fit the evolution of the parameters with the function $f=a x^{b}+c$, where the numerical values of the the parameters for the prestrain are: $a=-1.06 e-3 ; b=1.21 ; c=1.074$. And for $\kappa_{i}^{u b} \lambda_{0_{i}}^{u b}$ are: $a=5.582 ; b=1.437 ; c=132.2$; showing a good fitting of the relationship between the parameters. This shows that the exploring parameters can fall in a master relation with almost the same scaling exponent $b$ for the bundle pre-strain and for the stability of the chemical crosslinkers.

This effect is in agreement with the proposed model explained in the Figure 3 where the release of the trapped pre-stress drives the increase of the adhesion energy, and as a consequence increases the transition point. The Figure 8. $a$ plots the changes induced on $P_{u b}^{i}$, after each load cycle (computed using Eq. 11). It is observed that, as the number of cycles increases the probability $P_{u b}$, decreases for $\gamma=0.55$. The implications of this behavior are firstly that, the network structure stabilizes with a sufficient number of 


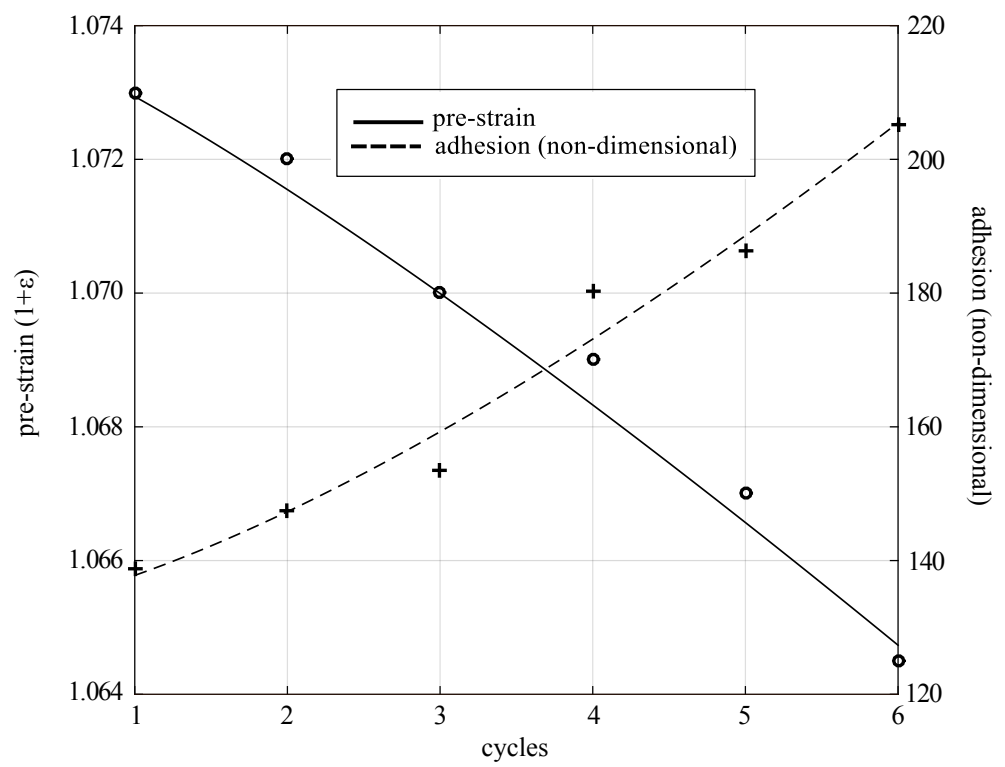

Figure 7: Evolution of the pre-strain $1+\epsilon$ and and the mesoscale crosslinkers adhesion $\kappa_{i}^{u b} \lambda_{0_{i}}^{u b}$

cycles, and secondly that the contribution with the contour length due to the reversible unbinding can be neglected.

Figure $8 . b$ shows the evolution of the contour length, $L_{c}$, as a function of the number of cycles. For the very first cycle, $L_{c}$ experiences the maximum increment due to the effect of reversible and irreversible cross-links rupture. As the number of cycles increases the unbinding probability, $P_{u b}$ decreases as does the contour length due to the reformation of reversible cross links, leading to an increment of the network stiffness in the large deformation range.

Figure 8.c shows the effect of cyclic hardening for a $\gamma_{\max }=0.55$ on the maximum shear stress, $\tau$, reached at the maximal cyclically applied strain $\gamma_{\max }$. The figure illustrates that for the first cycles the incremental rate in the reached shear stress is higher, evidencing more significant structural changes, but after some cycles the shear stress reaches a steady value. This evolution is also followed by significant changes in the shape of the stress-strain curve as shown in Figure 6. In terms of model parameters, it implies that the network structure does not evolve with subsequent cycles. Therefore, a stable elastic 


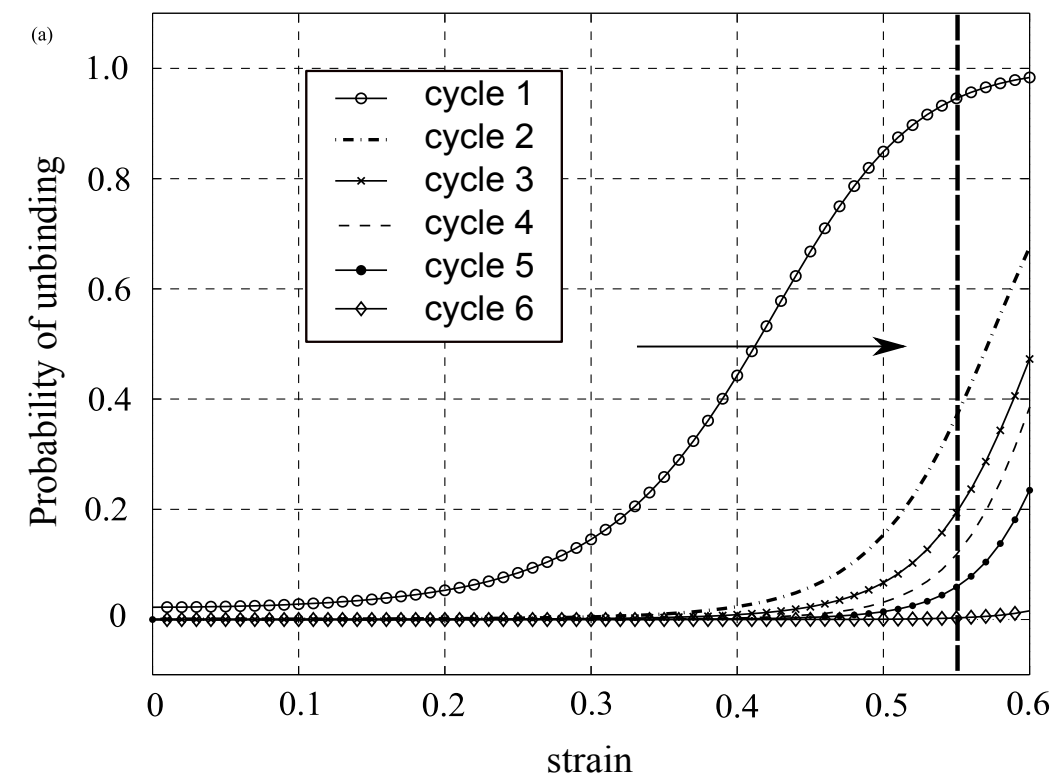

(b)

(c)
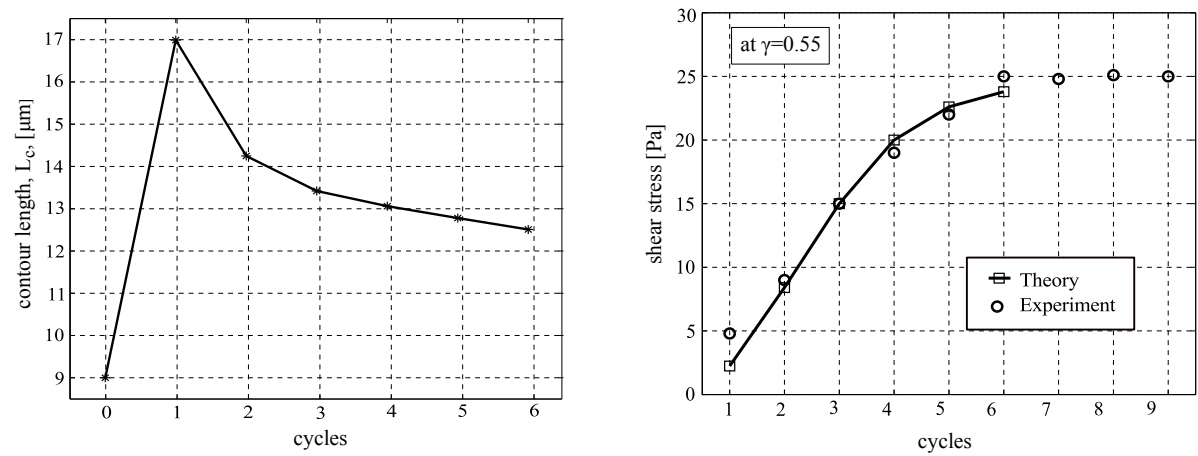

Figure 8: (a)Dependence among the unbinding probability $P_{u b}$ and strain $\gamma$ for different number of cycles. $(b)$ Evolution of the contour length as a function of the number of cycles. $(c)$ Difference between theoretical and experimental values of stress achieved at $\gamma=0.55$.

response is obtained between different cycles.

\section{Discussion and Conclusions}

This work proposes a mathematical model for explaining experimental studies conducted on reconstructed F-actin networks. The model is able to 
explain the observed effects of softening, when the network is working in a regime of monotonic loading, as well as for the cyclically stretch induced hardening. The softening effect experienced by the networks under monotonic loading is well documented for soft tissues and rubbers. On the contrary the strain induced cyclic hardening is not observed in other rubber-like materials and seems to be associated with this type of structures.

The proposed model relies on the worm-like chain model for semiflexible filaments which depends on three basic structural network parameter, i.e., filament contour length, $L_{c}$, filament persistent length, $l_{p}$, and the undeformed end-to-end filament distance, $r_{0}$. One of the key parameters in the state of the network is the value of $r_{0}$, which is a function of $L_{c}$ (see Eq. 6). If $L_{c}$ increases $r_{0}$ decreases. The intuitive image for the $w l c$ model is that the closer the $r$ value to $L_{c}$, the more nonlinear response of the filament and larger the tangent stiffness of the network. For bundles in networks with rigid crosslinkers, the stress on the filament will approach the locking point where the filament will break at $r \approx L_{c}$ and the network will collapse. In this regard, the proposed model introduces a split of the crosslink dynamics as chemical (reversible) and physical (irreversible) crosslink disruption that opens the possibilities of alternative dynamics which are able to reproduced the mechanical behavior of reconstituted F-actin networks observed in experiments.

Experimentally, it was observed that reconstituted F-actin networks crosslinked with $\alpha$-actinin showed an effect dependence of history, demonstrated by shearing to strains higher than $\gamma_{1}>\gamma_{0}$. As can be observed for a monotonic experiment where the network shows a stiffness region for strain values below of 0.25 and a softening response until strains of 1.2 , as we could observe in Figure 5. But if we consider the cyclic experiments where the $\gamma_{\max } 0.6$ all the cycles will have an asymptotic behavior for $\gamma \sim 0.6$ showing a clear dependence of the maximum strain value applied over the network.

In this model we consider that the physical crosslinkers will impose a double effect on the network. On one hand they pre-stresses the bundle, but on the other hand, the stretch of the chemical crosslinkers of $\alpha$-actinin (since we do not consider them as rigid cross links) will tilt the energy landscape of the crosslinkers toward a state of less adhesion energy. During the cyclic experiment, a certain amount of physical crosslinkers will break a consequently, the prestrain over the bundles and crosslinkers will decrease. Therefore the values of the $\Delta G^{\prime}$ or $\kappa^{u b} \lambda_{0}^{u b}$ will increase. As a consequence the probability of unbinding $P_{u b}$ decreases (see Figure 8b), and the contour length $L_{c}$ of the 
network will be less (see Figure 8c), showing an increase in the stiffening. The gelation state of the network changes from a weak gelation and high bundle prestrain towards a state of stable crosslikers with lower values of prestrain, which means a solid-like network.

The development of phenomenological model at mesoscale will helps the characterization of novel materials. Nevertheless, future works will be needed to improve the estimations used in the dynamics of crosslinkers in terms of the ratios between the concentration of F-actin and the concentration of $\alpha$ actin and the conditions of preparation of the network, in order to have an estimation of the tilt of energy landscape of $\alpha$-actinin which represents a very difficult task.

Yao et al. (2013) have reported dynamic nonlinearities in biopolymer networks consisting of filamentous actin cross-linked by $\alpha$-actinin- 4 . They observed that applied stress delays the onset of relaxation and flow, markedly enhancing gelation and extending the regime of solid-like behavior to much lower frequencies. They suggest that this macroscopic network response can be accounted for at the single molecule level by the increased binding affinity of the cross-linker under load, characteristics of the catch-bond-like behavior (Choi et al., 2005; Thomas et al., 2008; Zocchi, 2009). On the contrary our approach explains the increase of adhesion energy at the network scale, by means the interaction between physical and chemical crosslinkers and the relaxation of the trapped stress, due to the sacrificial bonds.

The model presented shows an alternative to extend the $w l c$ to describes the mechanical state of semiflexible networks with more complex gelation process by considering the dynamics of the crosslikers. At the same time, the proposed mesoscale model, within the framework of continuum mechanics, can be easily incorporated to computational simulations based on the finite element method, in order to consider more complex geometries. The effect introduced by the cyclic shear lead us to speculate on the role of molecular motors of actin-myosin in the cytoskeleton. Molecular motors are capable of applying cyclic strain to the bundle structure, helping to modify the internal prestress of the crosslinker protein structures. In this regard, it seems that the role of $\alpha$-actinin into the cytoskeleton structure could be more complex than just a rigid cross-linker. Additional experimental studies are required to better understand the interaction between molecular motors, crosslinkers, and actin filaments. However, the role of crosslinkers dynamics should be considered in future developments of constitutive models for cytoskeletonlike structures. 


\section{Acknowledgments}

The authors wish to thanks to the Dr. Kurt Schmoller and Prof.Dr Andreas Bausch from the Technical University of Munich (TUM) for sharing the raw data and comments from its experiments. H.L-M. thanks the Aragon's government and the University of Zaragoza for the FPI-DGA fellowship.

\section{Apendix}

\begin{tabular}{lll}
\hline $1+\epsilon$ & Bundle prestrain & 1.0730 \\
$n$ & Density of actin filaments & $9.6 e 19\left[\mathrm{~m}^{-3}\right]$ \\
$k_{B} T$ & Thermal energy & $4.1[\mathrm{pNn \textrm {m } ]}$ \\
$l_{p}$ & Persistence length & $17.48[\mu \mathrm{m}]$ \\
$L_{c}^{f}$ & Contour length & $10.5[\mu \mathrm{m}]$ \\
$L_{c}^{u b}$ & Contour length & $4.75[\mu \mathrm{m}]$ \\
$\lambda_{0}^{f}$ & Characteristic stretch irrev. crosslinkers & 1.024 \\
$\lambda_{0}^{u b}$ & Characteristic stretch rev. crosslinkers & 1.028 \\
$\kappa^{f}$ & Nondimensional irreversible crosslinkers stiffness & 7.1 \\
$\kappa^{u b}$ & Nondimensional reversible crosslinkers stiffness & 135 \\
\hline
\end{tabular}

Table 1: Model parameters for the monotonic experiment

\begin{tabular}{cccc}
\hline Cycle & $1+\epsilon_{i}$ & $\kappa_{i}^{u b}$ & $\lambda_{0_{i}}^{u b}$ \\
\hline 1 & 1.0730 & 135 & 1.028 \\
2 & 1.0720 & 140 & 1.053 \\
3 & 1.0700 & 145 & 1.059 \\
4 & 1.0690 & 170 & 1.061 \\
5 & 1.0670 & 175 & 1.065 \\
6 & 1.0645 & 190 & 1.080 \\
\hline
\end{tabular}

Table 2: Model parameters of the case of cyclic loading. Parameters $\kappa_{i}^{u b}$ and $\lambda_{0_{i}}^{u b}$ correspond to reversible cross linking occurring in the network under the action of cyclic loading. Parameter $1+\epsilon$ refers to the prestrain into the network.

\section{References}

A. Abhilash, P. Purohit, and S. Joshi. Stochastic rate-dependent elasticity and failure of soft fibrous networks. Soft Matter, 8(26):7004-7016, 2012. 
P. J. Arnoux, P. Chabrand, M. Jean, and J. Bonnoit. A visco-hyperelastic with damage for the knee ligaments under dynamic constraints. Computational Methods in Biomechanics and Biomedical Engineering, 5:167-174, 2002 .

E. Arruda and M. Boyce. A three-dimensional constitutive model for the large stretch behaviour of rubber elastic materials. Journal of the Mechanics and Physics of Solids, 41:389-412, 1993.

D. Balzani, J. Schröder, and D. Gross. Simulation of discontinuous damage incorporating residual stress in circumferentially overstretched atherosclerotic arteries. Acta Biomaterialia, 2:609-618, 2006.

G. Bell. Models for the specific adhesion of cells to cells. Science, 200(4342): 618-627, 1978.

K. Bertoldi and M. Boyce. Mechanics of the hysteretic large strain behavior of mussel byssus threads. Journal of Materials Science, 42(21):8943-8956, 2007.

A. Brown, R. Litvinov, D. Discher, P. Purohit, and J. Weisel. Multiscale mechanics of fibrin polymer: gel stretching with protein unfolding and loss of water. Science, 325(5941):741-4, 2009.

M. Buehler. Molecular nanomechanics of nascent bone: fibrillar toughening by mineralization. Nanotechnology, 18(29):295102, 2007.

B. Choi, G. Zocchi, S. Canale, Y. Wu, S. Chan, and L. Perry. Artificial Allosteric Control of Maltose Binding Protein. Physical Review Letters, 94 (3):7-10, 2005.

P. Ciarletta, P. Dario, and S. Micera. Pseudo-hyperelastic model of tendon hysteresis from adaptive recruitment of collagen type I fibrils. Biomaterials, 29(6):764-70, 2008.

C. Cyron, K. Müller, A. Bausch, and W. Wall. Micromechanical simulations of biopolymer networks with finite elements. Journal of Computational Physics, 244:236-251, 2013.

Pierre-Gilles De Gennes. Scaling concepts in polymer physics. Cornell university press, 1979 . 
Julie Diani, Bruno Fayolle, and Pierre Gilormini. A review on the mullins effect. European Polymer Journal, 45(3):601-612, 2009.

E. Ducrot, Y. Chen, M. Bulters, R. Sijbesma, and C. Creton. Toughening elastomers with sacrificial bonds and watching them break. Science, 344 (6180):186-189, 2014.

E. Evans. Probing the relation between force-lifetime-and chemistry in single molecular bonds. Annual review of biophysics and biomolecular structure, 30(1):105-128, 2001.

G. Fantner, T. Hassenkam, J. Kindt, J. Weaver, H. Birkedal, L. Pechenik, J. Cutroni, G. Cidade, G. Stucky, D. Morse, et al. Sacrificial bonds and hidden length dissipate energy as mineralized fibrils separate during bone fracture. Nature materials, 4(8):612-616, 2005.

M. Gardel, J. Shin, F. MacKintosh, L. Mahadevan, P. Matsudaira, and D. Weitz. Elastic behavior of cross-linked and bundled actin networks. Science, 304(5675):1301-5, 2004.

T. C. Gasser and G. A. Holzapfel. A rate-independent elastoplastic constitutive model for (biological) fiber-reinforced composites at finite strains: Continuum basis, algorithmic and finite element implementation. Computational mechanics, 29:340-360, 2002.

J. Golji, R. Collins, and M. Mofrad. Molecular mechanics of the $\alpha$-actinin rod domain: Bending, torsional, and extensional behavior. PLoS computational biolog, 5, 2009.

C. Heussinger, M. Bathe, and E. Frey. Statistical Mechanics of Semiflexible Bundles of Wormlike Polymer Chains. Physical Review Letters, 99(4):1-4, 2007.

J. Hokanson and S. Yazdami. A constitutive model of the artery with damage. Mechanical Research Communications, 24:151-159, 1997.

Gerhard A Holzapfel. Nonlinear solid mechanics, volume 24. Wiley Chichester, 2000.

Jonathon Howard. Mechanics of motor proteins and the cytoskeleton. Sinauer Associates Sunderland, MA, 2001. 
C. Hurschler, B. Loitz-Ramage, and R. Vanderby. A structurally based stressstrech relationship for tendon and ligament. Journal of Biomechanical Engineering, 119:392-399, 1997.

F. Keber, E. Loiseau, T. Sanchez, S. DeCamp, L. Giomi, M. Bowick, M. Marchetti, Z. Dogic, and A. Bausch. Topology and dynamics of active nematic vesicles. Science, 345(6201):1135-1139, 2014.

J. Kim and S. Sun. Continuum modeling of forces in growing viscoelastic cytoskeletal networks. Journal of theoretical biology, 256(4):596-606, 2009.

T. Kim, W. Hwang, H. Lee, and R. Kamm. Computational analysis of viscoelastic properties of crosslinked actin networks. PLoS computational biology, 5(7), 2009.

O. Lieleg, K. Schmoller, M. Claessens, and A. Bausch. Cytoskeletal polymer networks: viscoelastic properties are determined by the microscopic interaction potential of cross-links. Biophysical journal, 96(11):4725-32, 2009.

O. Lieleg, M. Claessens, and A. Bausch. Structure and dynamics of crosslinked actin networks. Soft Matter, 6(2):218, 2010.

O. Lieleg, J. Kayser, G. Brambilla, L. Cipelletti, and A. Bausch. Slow dynamics and internal stress relaxation in bundled cytoskeletal networks. Nature Materials, 10(3):236-242, 2011.

F. Mackintosh, J. Kas, and P. Janmey. Elasticity of semiflexible biopolymer networks. Physical Review Letters, 75:4425, 1995.

D. Mizuno, C. Tardin, C. Schmidt, and F. Mackintosh. Nonequilibrium mechanics of active cytoskeletal networks. Science, 315(5810):370-3, 2008.

J. Palmer and M. Boyce. Constitutive modeling of the stress-strain behavior of F-actin filament networks. Acta biomaterialia, 4(3):597-612, 2008.

Rob Phillips, Jane Kondev, and Julie Theriot. Physical Biology of the cell. Garland Science, 2009.

P Purohit, R Litvinov, A Brown, D Discher, and J Weisel. Protein unfolding accounts for the unusual mechanical behavior of fibrin networks. Acta biomaterialia, 7(6):2374-2383, 2011. 
J. F. Rodriguez, F. Cacho, J. A. Bea, and M. Doblare. A stochasticstructurally based three-dimensional finite-strain damage model for fibrous soft tissue. Journal of the Mechanics and Physics of Solids, 54:564-886, 2006 .

P. Saez, V. Alastrue, E. Peña, M. Doblare, and Martinez M. A. Anisotropic microsphere based approach to damage in soft fibered tissue. Biomechanics and modeling in mechanobiology, 11:595-608, 2012.

H. Schechtman and D. L Bader. Fatigue damage of human tendons. Journal of Biomechanics, 35:347-353, 2002.

K. Schmoller and A. Bausch. Similar nonlinear mechanical responses in hard and soft materials. Nature materials, 12(4):278-281, 2013.

K. Schmoller, O. Lieleg, and A. Bausch. Internal stress in kinetically trapped actin bundle networks. Soft Matter, 4(12):2365, 2008.

K. Schmoller, O. Lieleg, and A. Bausch. Structural and viscoelastic properties of actin/filamin networks: cross-linked versus bundled networks. Biophysical journal, 97(1):83-9, 2009.

K. Schmoller, P. Fernandez, R. Arevalo, D. Blair, and A. Bausch. Cyclic hardening in bundled actin networks. Nature Communications, 1:134, 2010.

S. Sun, S. Walcott, and C. Wolgemuth. Cytoskeletal cross-linking and bundling in motor-independent contraction. Current Biology, 20(15): R649-R654, 2010.

W. Thomas, V. Vogel, and E. Sokurenko. Biophysics of catch bonds. Annual review of biophysics, 37:399-416, 2008.

X. Trepat, L. Deng, S. An, D. Navajas, D. Tschumperlin, W. Gerthoffer, J. Butler, and J. Fredberg. Universal physical responses to stretch in the living cell. Nature, 447(7144):592-595, 2007.

T Van Dillen, P. Onck, and E. Van der Giessen. Models for stiffening in cross-linked biopolymer networks: A comparative study. Journal of the Mechanics and Physics of Solids, 56(6):2240-2264, 2008. 
S. Walcott and S. Sun. Active force generation in cross-linked filament bundles without motor proteins. Physical Review E - Statistical, Nonlinear and Soft Matter Physics, 82(5 Pt 1):050901, 2010.

Thomas A Witten and Philip A Pincus. Structured fluids. OUP, 2010.

L. Wolff, P. Fernandez, and K. Kroy. Inelastic mechanics of sticky biopolymer networks. New Journal of Physics, 12(5):053024, 2010.

L. Wolff, P. Fernández, and K. Kroy. Resolving the stiffening-softening paradox in cell mechanics. PloS one, 7(7):e40063, 2012.

N. Yao, C. Broedersz, M. Depken, D. Becker, M. Pollak, F. MacKintosh, and D. Weitz. Stress-enhanced gelation: A dynamic nonlinearity of elasticity. Physical Review Letters, 110(1):018103, 2013.

G. Zocchi. Controlling proteins through molecular springs. Annual review of biophysics, 38:75-88, 2009. 